\title{
Space Debris Removal under Spatial Grasp Technology
}

\author{
Peter Simon Sapaty ${ }^{1}$ \\ ${ }^{1}$ Institute of Mathematical Machines and Systems, National Academy of Sciences, Kiev, Ukraine \\ Correspondence: Peter Simon Sapaty, Institute of Mathematical Machines and Systems, National Academy of \\ Sciences, Kiev, Ukraine
}

Received: May 19, 2021 Accepted: June 18, 2021 Online Published: June 22, 2021

doi:10.5539/nct.v6n1p16 URL: https://doi.org/10.5539/nct.v6n1p16

\begin{abstract}
The threats of space debris are enormously high, which are increasing due to launch of multi-satellite constellations, especially in low-Earth orbit, with millions of pieces of junk there. Different passive and active debris removal methods are being developed like self-deorbiting of used satellites, drag sails, mechanical grasps, tethers and nets, also directed energy, lasers including. Space junk is the responsibility of the whole mankind, and the problem of managing space debris is both the international challenge and the opportunity to preserve the space environment for future space exploration missions. The paper shows how self-organized constellation networks of deorbiting satellites can organize multiple cleaning operations autonomously under the developed Spatial Grasp Technology (SGT), with cooperative involvement of the whole network and minimum interaction with costly ground antennas and stations. It also offers a unique solution where most dangerous junk items can themselves be treated as active virtual-physical items freely moving through terrestrial and celestial environments and ultimately finding, by their own initiative, the proper cleaning satellites. This can effectively organize the global junk management and removal problem, where the active junk items can keep initiative of self-removal for any time needed and using any distributed resources. A combined solution is also offered with initial global search for approximate satellite-debris matching, after which the junk is delegated its own initiative to find the absolute match by traveling around the globe as far and as long as required. The paper shows and explains different practical cleaning scenarios in the high-level Spatial Grasp Language (as key element of SGT) and possibilities of quick implementation of the approach.
\end{abstract}

Keywords: space debris, satellite constellations, Spatial Grasp Technology, active virtual-physical objects, self-cleaning junk scenarios, Spatial Grasp Language

\section{Introduction}

There are millions of pieces of space junk flying around the Earth, and especially in low-Earth orbits (LEO). Their amount may be rapidly increasing due to the intensive launch of multi-satellite constellations (sometimes even called "mega-constellations" for their sizes) for very different purposes and by different countries, particularly in LEO, especially when these satellites come to the end of service or collide with other satellites or the existing junk. The aim of this paper is to show how to deal with the space junk massively by using the very satellite constellations but oriented on specific cleaning applications (like de-orbiting the exiting junk to be burned in the atmosphere), which can be operating under the developed high-level Spatial Grasp model and related technology. Traditional parallel and distributed systems representations as parts or agents operating on individual algorithms and exchanging messages with each other are becoming inefficient and inadequate, as such systems can be difficult to outline as a whole ahead of the solutions needed on them, and this may especially relate to highly dynamic and rapidly growing systems around the Earth. Such systems need much stronger integration for pursuing global philosophy and rapidly changing goals. The current paper is oriented on a completely different paradigm for organization and management of large dynamic and distributed systems, which extends and transforms the traditional notion of algorithm for not only dealing with knowledge processing logic but also allowing it directly exist, propagate, develop and operate as an integral whole in any distributed spaces. Having self-spreading, self-replicating and self-recovering features resembling powerful world viruses, recent pandemics including, this ubiquitous Spatial Grasp model and technology is presented on methodological and implementation levels. The rest of the paper is organized as follows.

In Section 2 a summary on the debris problems and existing solutions is provided with mentioning such issues as legal questions of junk removal, debris surveillance and tracking, the removal complexity, already existing 
removal contracts and techniques, first removal missions, as well as some theoretical background for the removal. Section 3, starting from the simplest Spatial Grasp model explanation using a sort of spatial chart, briefs the main elements of Spatial Grasp Technology (SGT) and its high-level recursive Spatial Grasp Language (SGL), with already existing numerous publications, books including, on this approach, its implementation and numerous applications. This includes different types of distributed worlds SGT operates with, types of spatial variables of SGL, some of which may be stationary while others mobile, main types of SGL rules which can be nested, different control states provided by SGL scenarios propagation and used for global management, as well as general organization of the distributed and networked SGL interpreter. Section 4 shows how a special constellation of junk cleaning satellites can be converted under SGT into an intelligent team capable of organizing and executing massive debris removal operations with high autonomy and reduced ground communications. Section 5 shows in SGL how the debris discovery, general organization and execution scenarios can be expressed in SGL which is interpreted in a fully distributed way by the whole satellite cleaning network, with showing how the removal solutions can also be organized simultaneously for multiple debris. Section 6 provides a solution in SGL where the junk items can be virtually treated as active objects traveling around the Earth and finding proper cleaners by their own initiative, and this allows any time needed for finding a suitable match for such self-removal. It is also shown how this strategy can be effectively integrated with the one in the previous section. Section 7 concludes the paper with confirmation that the current SGT version can be readily implemented within standard environments, similar to the previous versions. It also mentions the future plans which include the new book currently in preparation.

\section{Debris Problems and Solutions}

\subsection{General on Debris}

The threats from existing numerous space debris are becoming enormously high (Space Debris; Teffer, 2018). The trash situation caused by intensive new launches and defunct satellites is getting worse and risks making space off-limits for future generations (Teffer, 2018) (see Figure 1 for some symbolic space junk pictures). Space junk is no one countries' responsibility (Space Debris, NASA Headquarters Library), but the responsibility of every space-faring country.

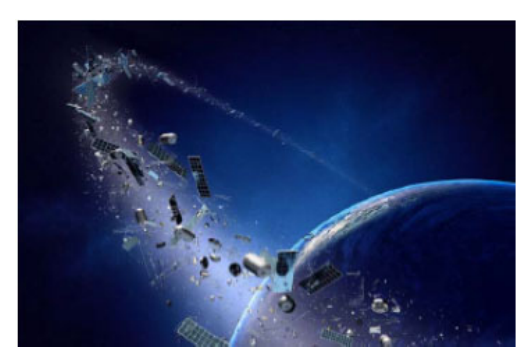

a

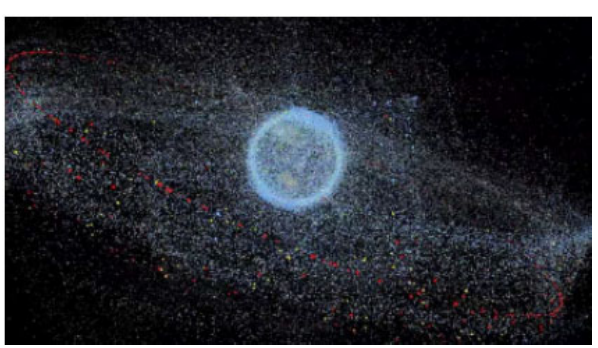

b

Figure 1. Space debris: (a) certain orbits related, (b) overall picture

The problem of managing space debris is both an international challenge and opportunity to preserve the space environment for future space exploration missions. LEO is an orbital space junk yard, with millions of pieces of space junk flying there. Most orbital debris comprises human-generated objects, such as pieces of space craft, tiny flecks of paint from a spacecraft, parts of rockets, satellites that are no longer working, or explosions of objects in orbit flying around in space at high speeds. The threats of space debris are increasing due to the launch of several multi-satellite constellations, particularly in low-Earth orbit. The new space paradigm and the increasing population of spacecraft in low-Earth orbit requires deorbiting systems that can satisfy space debris requirements (Deorbit Systems, National Aeronautics and Space Administration). Drag sails are the main technology, and several companies have already commercialized and sold these products. Other systems such as electromagnetic tethers, deployable booms, or the NASA Exo-brake have also already been prototyped and demonstrated in space.

\subsection{Legal Issues of Removal}

Various legal and political concepts to resolve the problem of the existing space debris in outer space are analyzed, also which measures to take to avoid space debris or to reduce potential space debris in the course of future space missions (Froehlich, 2019). From a scientific and technical point of view various studies are ongoing to analyze the feasibility of active debris removal. Nevertheless it has to be highlighted that outer space is an international area where various actors with different legal and political concepts are operating, a situation that leads to different 
approaches concerning such activities. Space debris is the global mounting ultimatum to the enduring maintainability of the Outer Space activities and it ought to be dealt in the very beginning, otherwise, it will be too late (Sheer \& Li, 2019). From couples of years ago, some incidents of collisions have enhanced the space debris accumulation, now crowded the corridor of earth orbit which constitutes the most serious pollutant of the near-earth space environment.

\subsection{Surveillance and Tracking}

Currently, over 22,000 objects larger than $10 \mathrm{~cm}$ are tracked by Space Surveillance Networks and recorded in their catalog to provide warnings to satellites in the path of these objects and to enable them to perform avoidance maneuvers (Aglietti, 2020). From a technical point of view, here the challenges are the identification, tracking, and cataloging of the centimeter-sized objects, still large enough to produce catastrophic damage but not included in the current catalogs. Radars have been the preferred ground-based system, in particular to monitor LEO, as they can operate independently day and night as well as in all meteorological conditions. However, most radar telescopes are optimized for astronomical observations rather than debris tracking and so bi-static systems have also been used to improve performance, and some have shown a capability to detect objects down to $1 \mathrm{~cm}$ at 100 $\mathrm{km}$. Similarly, systems combining laser ranging and passive optical tracking have been demonstrated to achieve good accuracy in determining the position of objects (within $10 \mathrm{~m}$ ). These capabilities and different organization catalogs have to come together to improve actionable knowledge of the orbital population.

\subsection{Complexity of Removal Problem}

Despite promising technology demonstrations, there is no one-size-fits-all solution for the growing problem of taking out the orbital trash (David, 2021). Even tiny pieces of space debris can have catastrophic effects. A Space Age "tragedy of the commons" is unfolding right under our nose (or, really, right over our head) and no consensus yet exists on how to stop it. For more than a half-century, humans have been hurling objects into low-Earth orbit in ever growing numbers. And with few meaningful limitations on further launches into that increasingly congested realm, the prevailing attitude has been persistently permissive: in orbit, it seems, there is always room for one more.

\subsection{Removal Contracts and Techniques}

"NASA and ESA studies show that the only way to stabilize the orbital environment is to actively remove large debris items (ESA commissions world's first space debris removal, ESA /Safety \& Security/Clean Space; Parsonson, 2020; Humza, 2020). ClearSpace-1 will be the first space mission to remove an item of debris from orbit, planned for launch in 2025 , as the first-ever space mission to clean orbital junk with the use of a giant claw. The first-of-its-kind mission is not only new in terms of what it's setting out to achieve, but also represents a shift in strategy for the ESA, which has chosen a private firm to design and engineer its own spacecraft and plan of execution. A Japanese company said recently it will develop a satellite to clean up floating space debris by using laser beams, with the aim of starting the service in 2026 (Japanese company planning space debris removal by laser on satellite, 2020). Satellite communications company Sky Perfect JSAT Corp. said the project will be the first to use laser beams to remove space debris such as defunct satellites and rocket sections. To preserve a secure space environment, the active removal or de-orbiting of space debris is an emergent technological challenge. If remedial action is not taken in the near future it will be difficult to sustain human space activities. To overcome this issue, several other methods for the removal and de-orbiting of debris have been proposed so far; classified as either contact (e.g., robotic arm, tether net, electrodynamic tether) or contactless ones like plasma beam (Plasma thruster: New space debris removal technology, 2018) ejected from the satellite to impart a force to the debris thereby decelerating it, which results in it falling to a lower altitude, re-entering the Earth's atmosphere and burning up naturally (see Figure 2 for some existing junk cleaning techniques).

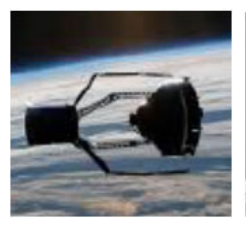

a

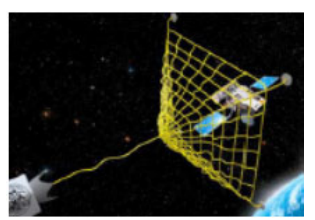

b

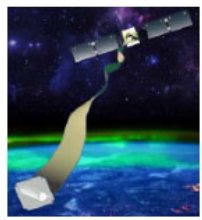

C

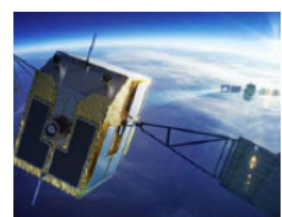

d

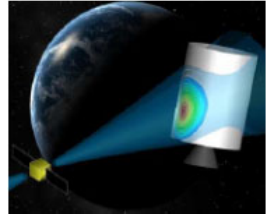

e

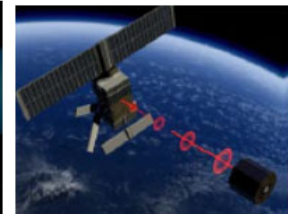

f

Figure 2. Active de-orbiting techniques: (a) mechanical grasp, (b) net, (c) tether, (d) laser, (e) ion beam, (f) directed energy 


\subsection{Very First Missions}

The spacecraft and the 17-kilogram dummy satellite (the debris to be cleaned up) will separate and then perform a high-stakes game of cat and mouse (Hunt, 2021; Obe, 2021; Weiner, 2021). A demonstration mission to test new technology developed by the company Astroscale to clean up space debris is to be launched from the Baikonur Cosmodrome in Kazakhstan. Known as ELSA-d, the mission will exhibit technology that could help capture space junk, the millions of pieces of orbital debris that float above Earth. The more than 8,000 metric tons of debris threaten the loss of services we rely on for Earth-bound life, including weather forecasting, telecommunications and GPS systems.

\subsection{Some Theoretical Background}

To reduce the rising influence of the space debris and improve the safe performance of the space mission, the three-stage removal strategy for space debris is proposed (Y. Chen, Bai, Zhao, Wang, \& X. Chen, 2020). Firstly, the multiple spacecrafts including one main spacecraft and some following spacecraft for space debris removal mission is considered. Then, the fuel, time and the quantity of the following spacecraft are defined as the constraints. Moreover, using the minimum fuel consumption as the optimal object, the mathematical model of the debris removal problem is established. Finally, the genetic algorithm is applied to solve this problem. The proposed three-stage space debris removal can effectively reduce the fuel consumption. Numerical simulation verifies the effectiveness of the proposed space debris removal scheme. In (Klima et al., 2018), space debris removal from a game-theoretic perspective is studied, focused on the question whether and how self-interested agents can cooperate. Centralized and decentralized solutions are compared. In addition it was investigated whether agents can learn optimal strategies by reinforcement learning, and for this an orbital simulator was used. Studied were both single- and multi-agent approaches using stochastic (Markov) games and reinforcement learning. The main finding was that the cost of a decentralized, competitive solution can be significant, which should be taken into consideration when forming debris removal strategies.

\section{Spatial Grasp Model and Technology (SGT)}

\subsection{The Simplest SG Model Explanation}

SGT operates by spatial scenarios self-spreading in physical and virtual worlds while creating. matching, transforming and managing them. In the simplest case, such a scenario is shown in Figure 3, consisting of following each other parts or grasps Gi of arbitrary complexity, with the next grasps developing in parallel from positions in space reached by the previous grasps. The interpreted scenario text is not staying in any fixed point or points in space but rather spreads itself while carrying further its remainder and omitting the utilized parts.

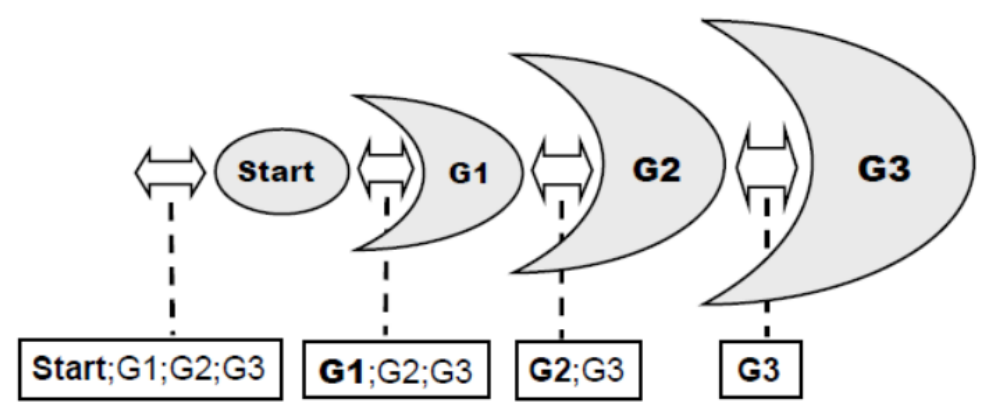

Figure 3. Spatial Grasp Model elementary explanation

In general, the model is much more advanced and complex, with capability of returning the obtained results and states whatever remote and multiple they might appear. It also allows to make any decisions for the further space navigation, creates dynamic operational infrastructures capable of solving any distributed problems, also effectively mimicking or implementing any other models (Petri nets and neural nets including), and so on.

\subsection{The Spatial Grasp Language}

The mentioned above and many other SG model's capabilities can be expressed by the recursive high level Spatial Grasp Language (SGL) in which all spatial scenarios are represented, with its top level syntax following. (The overall SGL scenario is called grasp, syntactic categories are shown in italics, vertical bar separates alternatives, 
parts in braces indicate zero or more repetitions with a delimiter at the right if multiple, and constructs in brackets are optional.)

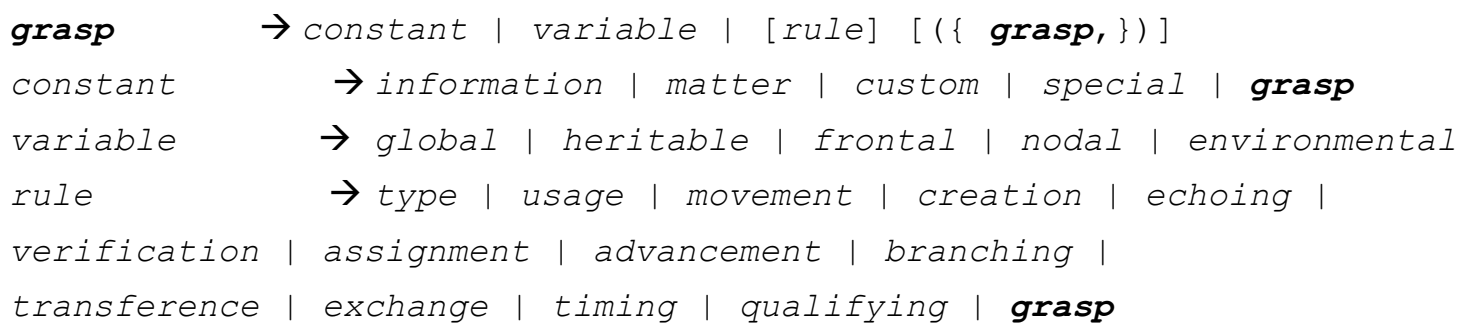

\subsection{The Worlds SGT Operates with}

SGT allows us to directly operate with the following world representations: Physical World (PW), considered as continuous and infinite, where each point can be identified and accessed by physical coordinates; Virtual World (VW), which is discrete and consists of nodes and semantic links between them; and Executive world (EW) consisting of active "doers" with communication possibilities between them. Different kinds of combination of these worlds can also be possible within the same formalism, as follows: Virtual-Physical World (VPW) where individually named VW nodes can associate with coordinates of certain PW points or any its regions; Virtual-Execution World (VEW), where doer nodes may have special names assigned to them and semantic relations in between, similarly to pure VW nodes; Execution-Physical World (EPW) can have doer nodes associated with certain PW coordinates; and Virtual-Execution-Physical World (VEPW) combining all features of the previous cases.

\subsection{SGL Variables}

Spatial variables, stationary or mobile, which can be used in fully distributed physical, virtual or executive environments, are effectively serving multiple cooperative processes under the unified control. These are: Global variables (most expensive), which can serve any SGL scenarios and be shared by them, also by their different branches; Heritable variables appearing within a scenario step and serving all subsequent, descendent steps; Frontal variables serving and accompanying the scenario evolution, being transferred between subsequent steps; Environmental variables allowing us to access, analyze, and possibly change different features of physical, virtual and executive words during their navigation; and finally, Nodal variables as a property of the world positions reached by scenarios and shared with other scenarios in same positions.

\subsection{SGL Rules}

SGL rules, capable of representing any actions or decisions, belong to the following main categories: (a) hierarchical fusion and return of potentially remote data; (b) distributed control, sequential and/or parallel, in both breadth and depth of the scenario evolution; (c) a variety of special contexts detailing navigation in space, also clarifying character and peculiarities of the embraced operations and decisions; (d) type and sense of a value or its chosen usage for guiding automatic language interpretation; and (e) individual or massive creation, modification, or removal of nodes and connecting links in distributed knowledge networks, allowing us to effectively work with arbitrary knowledge structures. All rules are pursuing the same unified ideology and organizational scheme, as follows: (1) they start from a certain world position, being initially linked to it; (2) perform or control the needed operations in a distributed space, which may be branching, stepwise, parallel and arbitrarily complex, also local and remote; and (3) produce or supervise concluding results of the scenario embraced, expressed by control states and values in different points.

\subsection{Control States}

The following control states can appear after completion of different scenario steps. Indicating local progress or failure, they can be used for effective control of multiple distributed processes with proper decisions at different levels. These states are: thru - reflects full success of the current scenario branch with capability of further development; done - indicates success of the current scenario step with its planned termination; fail - indicates non-revocable failure of the current branch and no possibility of further development from the location reached; and fatal - reporting terminal failure with nonlocal effect, while triggering massive abortion of all currently evolving scenario processes and removal of associated temporary data with them. These control states, appearing in different branches of parallel and distributed scenario at bottom levels, can be used to obtain generalized control 
states at higher levels, up to the whole scenario, in order to make proper decisions for the further scenario evolution.

\subsection{Networked SGL Interpreter}

Communicating Interpreters of SGL can be in arbitrary number of copies, say, up to millions and billions, which can be effectively integrated with any existing systems and communications, and their dynamic networks can represent powerful spatial engines capable of solving any problems in terrestrial and celestial environments. Such collective engines can simultaneously execute many cooperative or competitive tasks without any central resources or control, as symbolically depicted in Figure 4SGL interpreters just named U, as universal computational and management nodes).

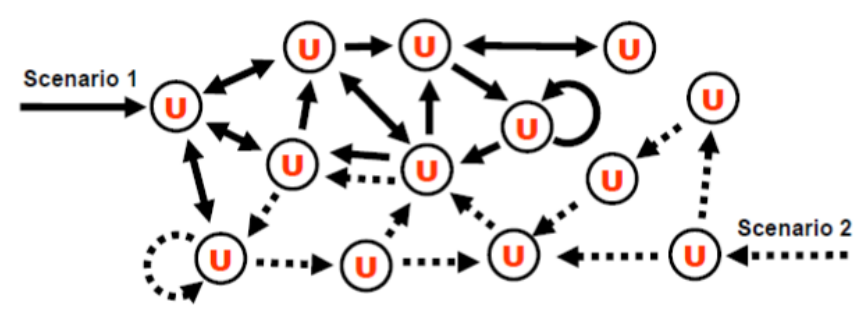

Figure 4 SGL interpretation networks as a global world computer

As both backbone and nerve system of the distributed interpreter, its hierarchical spatial track system dynamically spans the worlds in which SGL scenarios evolve, providing automatic control of multiple distributed processes. Self-optimizing in parallel echo processes, this (generally forest-like) distributed structure provides hierarchical command and control, also remote data and code access. It supports spatial variables and merges distributed control states for making proper decisions at different organizational levels. The track infrastructure is automatically distributed between different active components (humans, robots, computers, smartphones, satellites, etc.) during scenario spreading in distributed environments.

Detailed information on SGT, SGL and its networked interpreter, also solving numerous problems from very different classes under such approach, can be obtained from many existing publications, including (Sapaty, 1999; 2005; 2017; 2019a; 2019b; 2020; 2021; n.d.), also just by spatial grasp in google.com.

\section{Itelligent Constellation of Junk Cleaners}

In (Sapaty, 2021a; 2021b) organization of constellations of small satellites was considered under SGT with their applications for very different purposes, where large satellite groups can become intelligent self-organized systems capable of solving very complex problems autonomously, also with reduced engagement of costly ground antennas and their infrastructures. Dealing with such complex problem as huge amount of space debris can also be possible only by using large constellations, even mega-ones, of special cleaning (like de-orbiting) satellites working together as a global goal-oriented system. In Figure 5, such constellation of cleaners is symbolically shown with all units supplied with mechanical grasps, which may also be equipped with any other techniques (including the ones shown in Figure 2). 


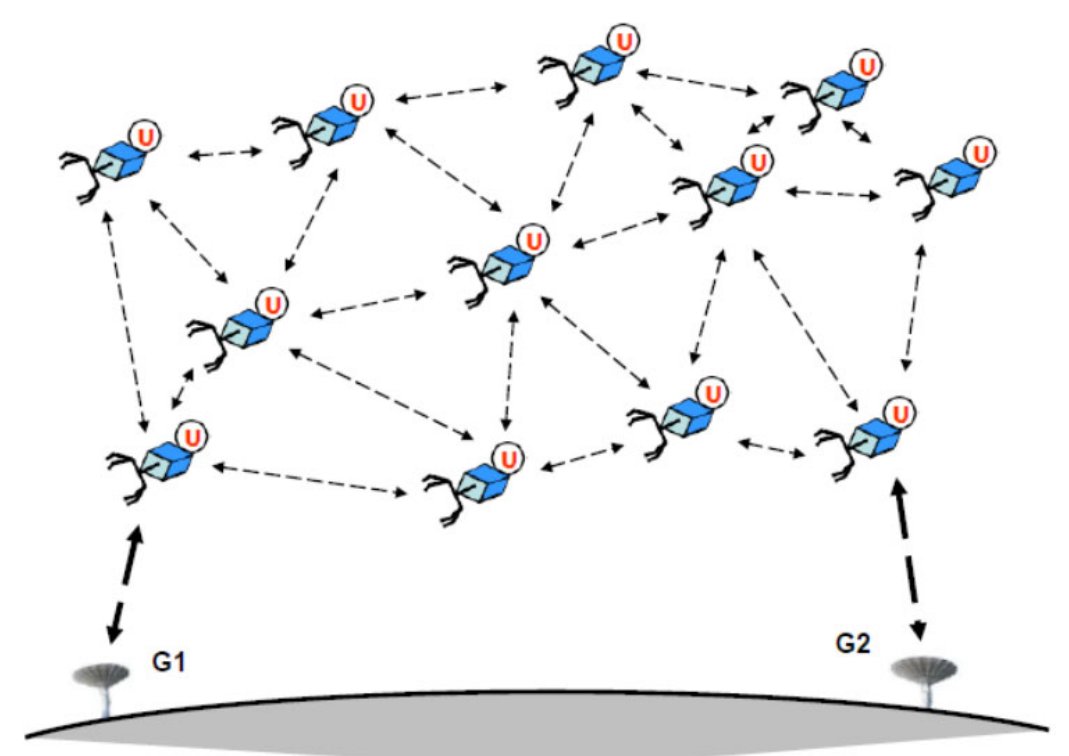

Figure 5. Converting constellation of junk cleaners into self-organized system

In the following sections we will be showing and discussing some elementary scenarios in SGL for cooperating cleaning procedures executed by such satellites groups.

\section{Junk Removal by Initiative of Cleaners Network}

\subsection{Finding and Deorbiting of a Single Junk Item}

It is supposed that ground station G2 using radar discovers a suitable junk item and records its parameters (incl. current time, location, size, expected orbit, etc.) in frontal variable Details, and then transfers this to the nearest satellite-cleaner C1, by the radar too, as in the following SGL scenario. (See also Figure 6, with the related debris item named as D1.) The chosen satellite starts repetitive and parallel constellation flooding and coverage (up to the whole network) using direct links between satellites until finding the most suitable cleaner for the junk deorbiting (i.e. its Snapshot of the nearest satellite matches Details). If deorbiting provided successfully, the further constellation coverage is aborted with appropriate cleaning of the network (operation abort). Otherwise, the network search will continue finding of the appropriate cleaner. As the network search takes time, with satellites constantly moving, and the junk item moving too, the networked coverage scenario regularly updates the expected for this moment of time (with use of global variable TIME) junk parameters in the moving variable Details. Operations hop_first navigate the cleaners network with blocking possible cycling (using nodes marking on the internal interpretation level).

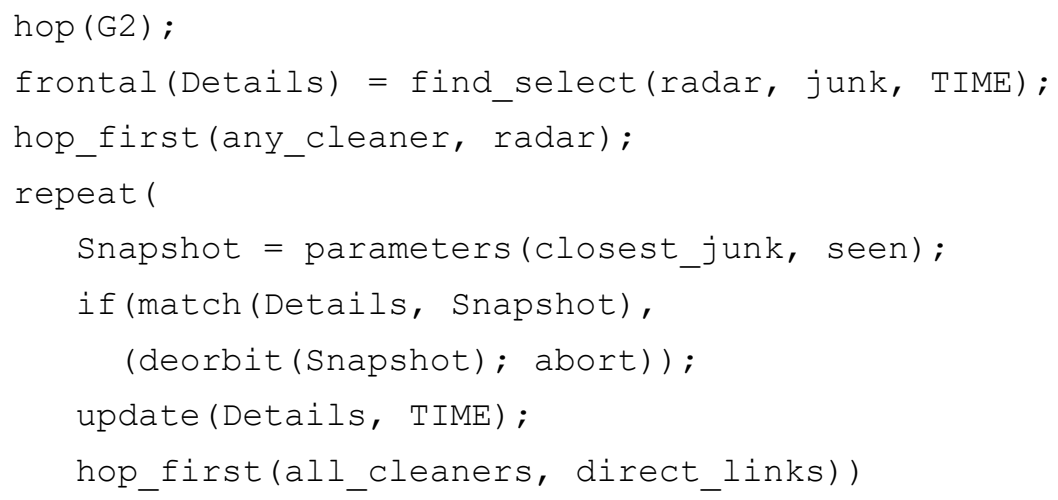




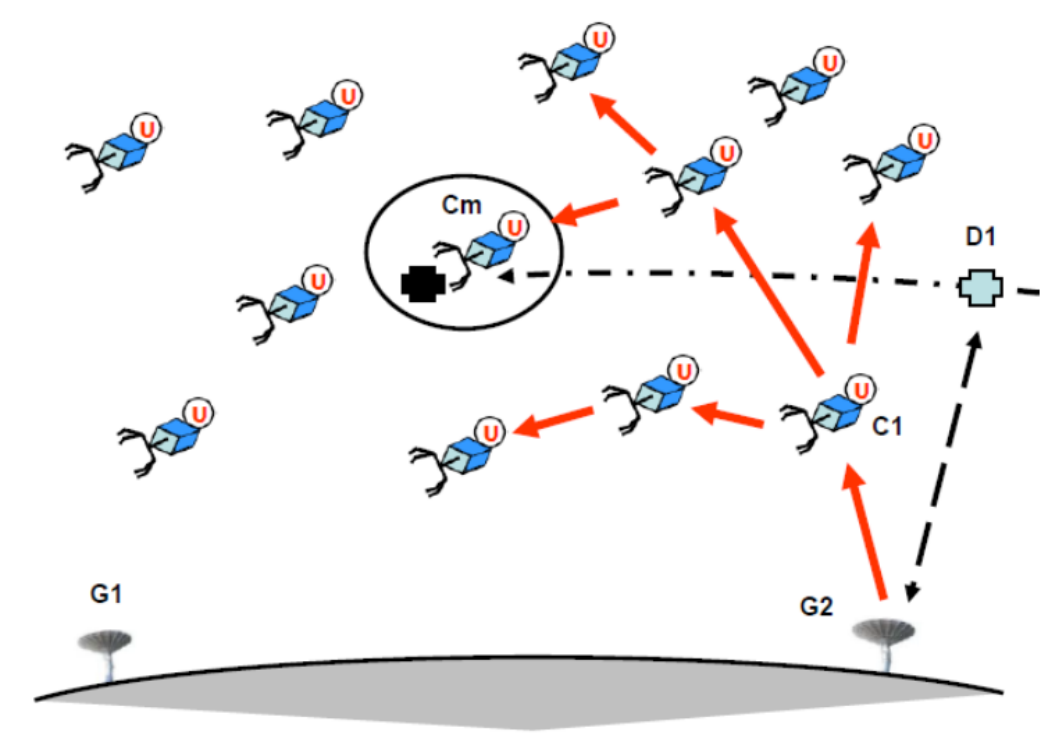

Figure 6. Autonomous reaching of the junk needed by self-organized network of cleaners

\subsection{Removing Many Junk Items Simultaneously}

Any number of registered junk items can be found by the ground stations with launching deorbiting processes simultaneously, as by the following scenario (see also Figure 7) using two ground stations G1 and G2, each trying to find and remove suitable junks independently by the same network of cleaners. This navigation independence within the same satellite network is guaranteed by different navigation colors using frontal environmental variables COLOR (here corresponding to names G1 and G2), which also influence the names of used SGL variables and internal blocking of cycling. In a further development, each ground station can also detect and record any number of suitable junk items for the removal attempts (and not only a single one for each station, as below, shown in Figure 7 as D1 and D2), which can trigger independent network navigation with individual colors for each junk item too.

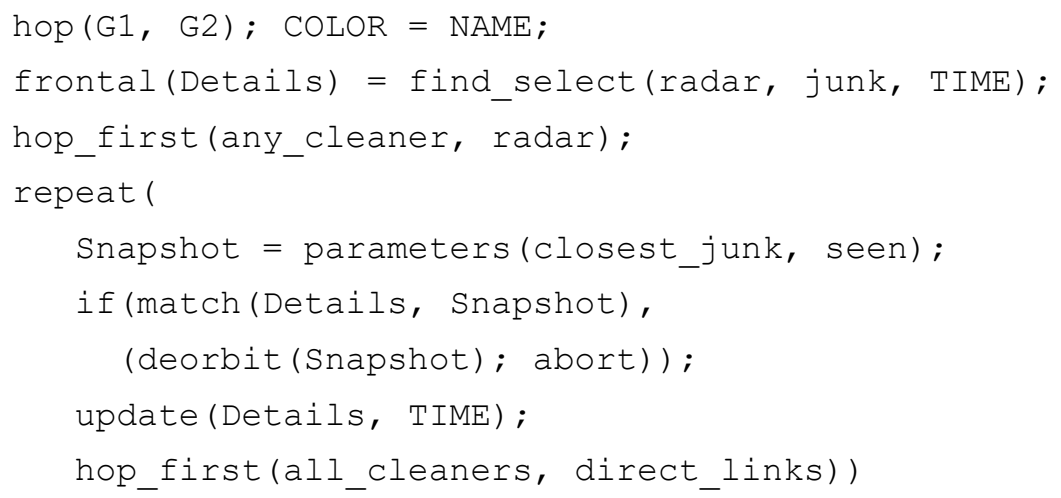




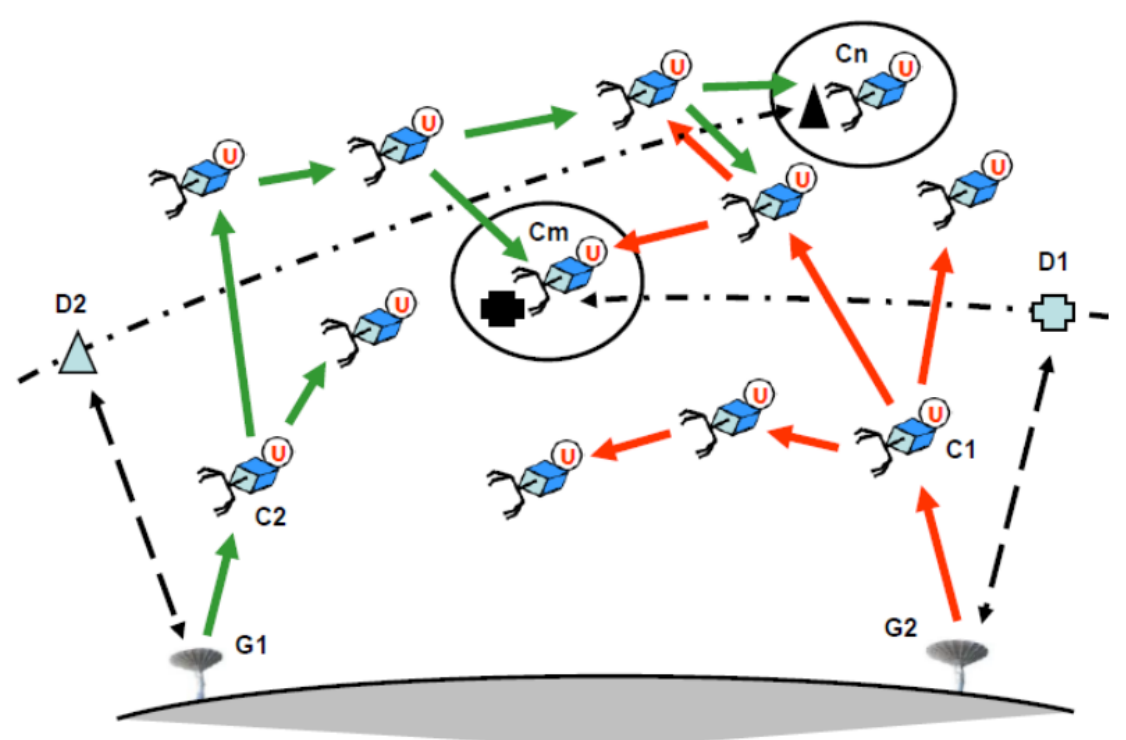

Figure 7. Simultaneous junk reaching and removal

\section{Active Virtual Junk Solution}

\subsection{Self-Removing Junk Model}

In the previous section we used parallel network search for a possible match between the selected junk and the cleaning satellite which happened to be close enough to perform deorbiting operation. If the latter unsuccessful, the parallel network flooding would be continued until full network coverage, which may not always guarantee the final success as satellites and junk may happen to be far away from each other during this period of time, on different orbits, and moving in different directions. The following scenario (see also Figure 8) is putting the global search for the match not on the initiative of the cleaners network but on the activity of the junk item itself by having represented it as a virtual identity process traveling between distributed cleaners any needed time, including endlessly, with possibility of entering same cleaners many times unless one of them happens to match the needed junk item at some moment of time. And such virtual junk can be travelling only via local neighbors which appear more suitable for the continuing search, thus without expensive parallel flooding of the whole network. It is supposed from the beginning that the Details of the junk item of interest are obtained by some ground station, which sends them directly to the discovered nearest cleaner $\mathrm{C} 1$, from which the virtual junk identity process begins traveling autonomously through the cleaners constellation, as long as needed, and around the Earth.

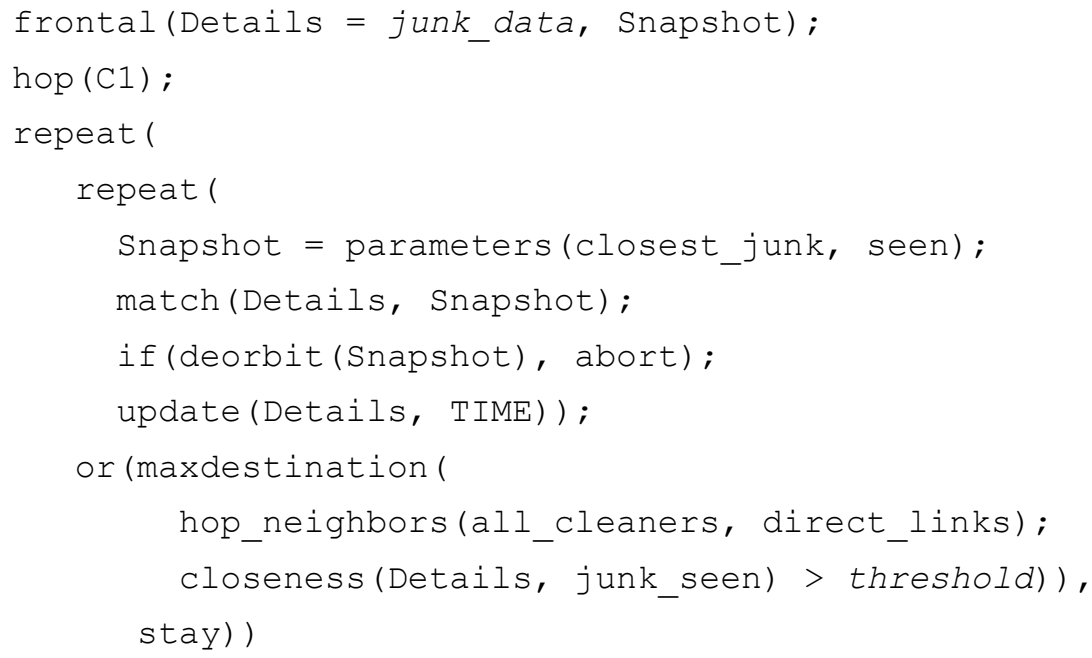




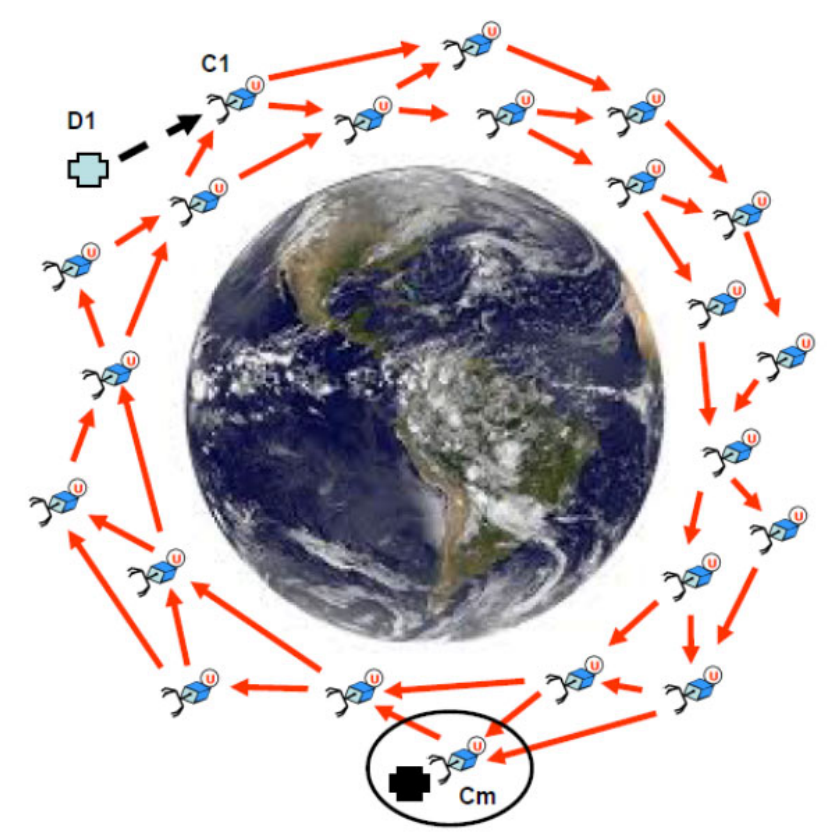

Figure 8. Active virtual junk self-searching for its removal

\subsection{Combined Organization}

It may be useful to combine the two different strategies discussed above where first one was the matching search on the initiative and activity of the whole cleaners network by its global self-flooding, and the other was unlimited localized search for the appropriate cleaner by the initiative of virtual junk itself. The following scenario combines the global search for initial approximate matching (similar to Figures 6, 7), starting from some ground station G2, after which the junk is delegated its own initiative to find absolute match with any time needed for this (as in Figure 8). In a further development we can use such symbiosis for initial discovery of any junk items, their approximate matching with cleaners, and then converting to active virtual junk items themselves traveling and finding the final solutions.

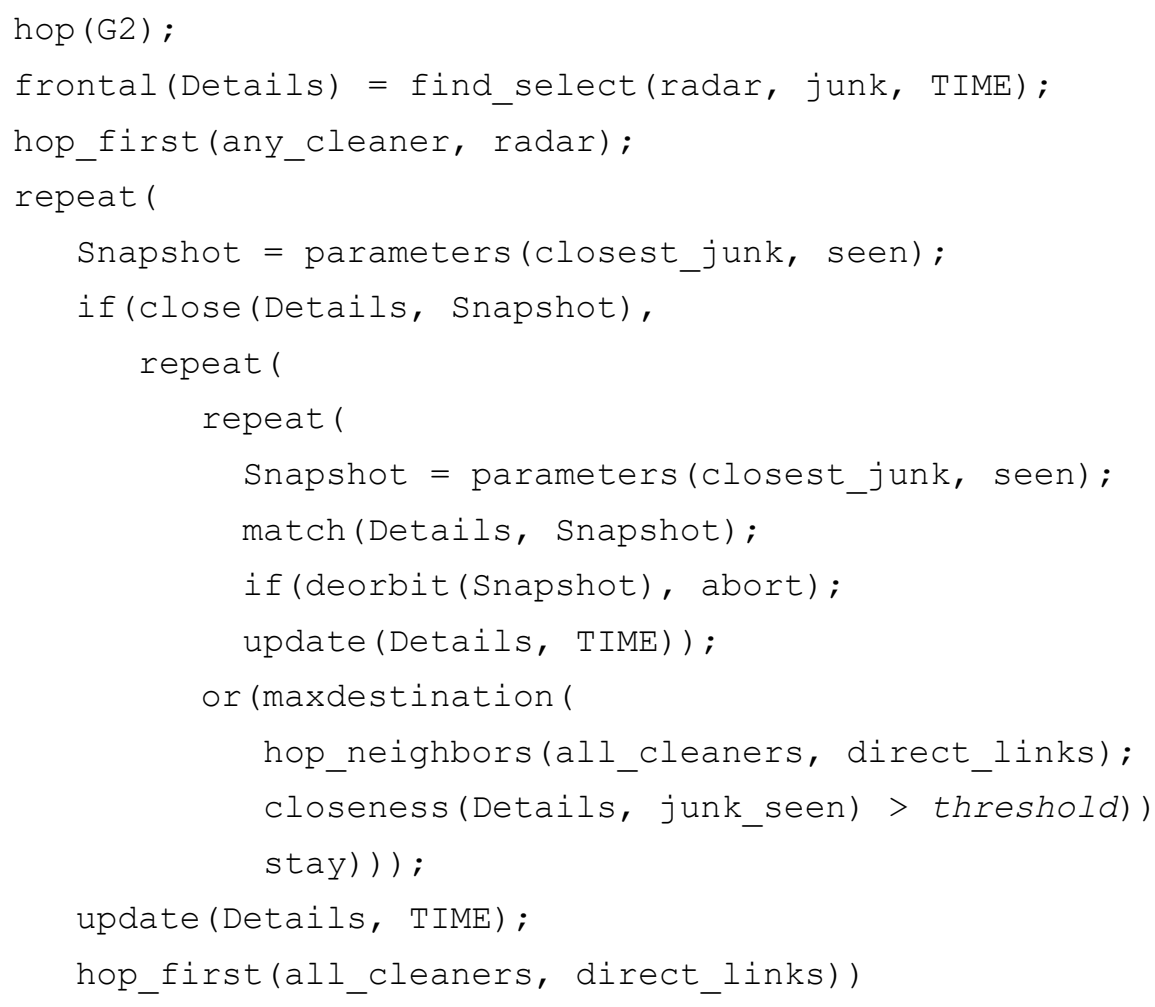




\section{Conclusions}

We have considered different possibilities of organization of massive removal of most dangerous junk items, especially in the LEO orbits, by special large constellations of cleaning satellites, which can be effectively organized as intelligent autonomous systems under the developed Spatial Grasp Model and Technology. This approach, instead of representing distributed systems as collection of parts or agents exchanging messages, which may often be difficult of not impossible to organized in highly dynamic and crisis situations, allows for such systems integral, holistic and semantic level solutions in the form of self-evolving and self-matching spatial patterns, which can often simplify and shorten global management code up to a hundred times in comparison to other approaches and languages. Of course, such mega-cleaning constellations do not exist yet, and unfortunately many private companies and governmental organizations are chaotically launching numerous and cheap satellites (which soon will count up to 100 thousand in LEO, Sapaty, 2021b). But the danger of uncontrolled rubbishing of space around Earth may be very high and lead to even more severe consequences than the global warming and the current worldwide covid disaster. So we hope that this article may be stimulating and useful ahead of creating global cleaning approaches, and we also plan to offer and discuss these issues with reputable international organizations, UN including. We plan to investigate further different techniques for collective removal of space junk under SGT and SGL with another publications planned, new book including, which is currently in progress (Sapaty, 2021c). Based on our experience of implementing the previous SGT versions in different countries, within traditional university environments including, we can ensure that the basics of the current technology version can also be implemented by a group of system programmers within 4-5 months, and if needed, with the ready assistance of the current author, as before.

\section{References}

Aglietti, G. S. (2020). From Space Debris to NEO, Some of the Major Challenges for the Space Sector. Front. Space Technol, 1(2). https://doi.org/10.3389/frspt.2020.00002

Chen, Y., Bai, Y., Zhao, Y., Wang, Y., \& Chen, X. (2020). Optimal mission planning of active space debris removal based on genetic algorithm. In IOP Conference Series: Materials Science and Engineering (Vol. 715, No. 1, p. 012025). IOP Publishing. https://doi.org/10.1088/1757-899X/715/1/012025

David, L. (2021). Space Junk Removal Is Not Going Smoothly, Scientific American. Retrieved April 14, 2021, from https:/www.scientificamerican.com/article/space-junk-removal-is-not-going-smoothly/

Deorbit Systems, National Aeronautics and Space Administration. Retrieved Nov 28, 2020, from https://www.nasa.gov/smallsat-institute/sst-soa-2020/passive-deorbit-systems

ESA commissions world's first space debris removal, ESA /Safety \& Security/Clean Space. Retrieved December 9, 2019 ,

from https://www.esa.int/Safety_Security/Clean_Space/ESA_commissions_world_s_first_space_debris_removal

Froehlich, A. (Ed.) (2019). Space Security and Legal Aspects of Active Debris Removal. Springer. https://doi.org/10.1007/978-3-319-90338-5

Humza. (2020). The first-ever space mission to clean orbital junk will use a giant claw. Techspot, Dec 1, 2020.

Hunt, K. (2021). Mission to clean up space junk with magnets set for launch. CNN. Retrieved April 1, 2021, from https://edition.cnn.com/2021/03/19/business/space-junk-mission-astroscale-scn/index.html

Japanese company planning space debris removal by laser on satellite. KYODO NEWS. Retrieved Aug 8, 2020, from

https://english.kyodonews.net/news/2020/08/fc06829d1d9a-japanese-company-planning-space-debris-remo val-by-laser-on-satellite.html

Klima, R., Bloembergen, D., Savani, R., Tuyls, K., Wittig, A., Sapera, A., \& Izzo, D. (2018). Space debris removal: Learning to cooperate and the price of anarchy. Frontiers in Robotics and AI, 5, 54. https://doi.org/10.3389/frobt.2018.00054

Obe, M. (2021). Japan's Astroscale launches space debris-removal satellite. Nikkei Asia. Retrieved March 22, 2021 , https://asia.nikkei.com/Business/Aerospace-Defense/Japan-s-Astroscale-launches-space-debris-removal-sat ellite

Parsonson, A. (2020). ESA signs contract for first space debris removal mission. Space News, Dec 2, 2020. Retrieved from https://spacenews.com/clearspace-contract-signed/ 
Plasma thruster: New space debris removal technology. (2018). TOHOKU UNIVERSITY. Retrieved 27 Sep, 2018, from https://www.eurekalert.org/pub_releases/2018-09/tu-ptn092718.php

Retrieved from https://www.techspot.com/community/topics/the-first-ever-space-mission-to-clean-orbital-junk-will-use-a-g iant-claw.266509/

Sapaty, P. (1999). Mobile processing in distributed and open environments (Vol. 10, p. 410). John Wiley \& Sons.

Sapaty, P. (2005). Ruling distributed dynamic worlds (Vol. 65, P. 255). John Wiley \& Sons. https://doi.org/10.1002/0471656356

Sapaty, P. (2021a). Managing multiple satellite architectures by spatial grasp technology. Mathematical machines and systems, N1, 3-16. https://doi.org/10.34121/1028-9763-2021-1-3-16

Sapaty, P. (2021b).Spatial Management of Large Constellations of Small Satellites. Mathematical machines and systems, $\mathrm{N} 2$.

Sapaty, P. (2021c). Spatial Grasp as a Model for Space-based Control and Management Systems. Mathematical $\begin{array}{llrll}\text { machines } \quad \text { and } & \mathrm{N} 1, & \text { Retrieved } & \text { from }\end{array}$ http://www.immsp.kiev.ua/publications/articles/2021/2021_1/Sapaty_book_1_2021.pdf

Sapaty, P. (n.d.). A Distributed Processing System: European Patent. № 0389655. Publ, 10(93), 40.

Sapaty, P. S. (2017). Managing distributed dynamic systems with spatial grasp technology. Springer International Publishing. https://doi.org/10.1007/978-3-319-50461-2

Sapaty, P. S. (2019a). Complexity in International Security: A Holistic Spatial Approach (p. 160). Emerald Publishing. https://doi.org/10.1108/9781789737158

Sapaty, P. S. (2019b). Holistic analysis and management of distributed social systems (p. 234). Springer. https://doi.org/10.1007/978-3-030-01830-6

Sapaty, P. S. (2020). Global Network Management under Spatial Grasp Paradigm. Global Journal of Research In Engineering, 58-69. $\quad$ Retrieved from https:/engineeringresearch.org/index.php/GJRE/article/view/2082/2013

Sapaty, P. S. (2021). Symbiosis of Real and Simulated Worlds under Spatial Grasp Technology (p. 251). Springer, 2021. https://doi.org/10.1007/978-3-030-68341-2

Sheer, A., \& Li, S. (2019). Space Debris Mounting Global Menace Legal Issues Pertaining to Space Debris Removal: Ought to Revamp Existing Space Law Regime. Beijing L. Rev., $10,423$. https://doi.org/10.4236/blr.2019.103025

Space Debris, NASA Headquarters Library. Retrieved from https://www.nasa.gov/centers/hq/library/find/bibliographies/space_debris

Space Debris. Retrieved from https://en.wikipedia.org/wiki/Space_debris

Teffer, P. (2018). Europe's space trash chief: situation getting worse, Interview. EUOBSERVER, Aug. 2018. Retrieved from https://euobserver.com/science/142685

Weiner, C. (2021). New Effort to Clean Up Space Junk Reaches Orbit. Retrieved March 21, 2021, from https:/www.npr.org/2021/03/21/979815691/new-effort-to-clean-up-space-junk-prepares-to-launch

\section{Copyrights}

Copyright for this article is retained by the author(s), with first publication rights granted to the journal.

This is an open-access article distributed under the terms and conditions of the Creative Commons Attribution license (http://creativecommons.org/licenses/by/4.0/). 